Muschalla, B., \& Linden, M. (2011). Sozialmedizinische Aspekte bei psychischen Erkrankungen. Teil 2: Psychische Erkrankungen im medizinischen Versorgungssystem und therapeutische Maßnahmen. Der Nervenarzt, 82, 1187-1200.

\title{
Sozialmedizinische Aspekte bei psychischen Erkrankungen
}

Teil 2: Psychische Erkrankungen im medizinischen Versorgungssystem und therapeutische Maßnahmen

Beate Muschalla, Michael Linden

Forschungsgruppe Psychosomatische Rehabilitation an der Charité Universitätsmedizin Berlin und Abteilung Verhaltenstherapie und Psychosomatik am Rehabilitationszentrum Seehof der Deutschen Rentenversicherung Bund, Teltow/Berlin

Fortbildungsartikel zur Publikation in: Der Nervenarzt

Anschrift der Autoren:

Prof. Dr. M. Linden

Forschungsgruppe Psychosomatische Rehabilitation

Rehabilitationsklinik Seehof der Deutschen Rentenversicherung Bund

Lichterfelder Allee 55

14513 Teltow/Berlin

email: michael.linden@charite.de

Wir danken Hr. Dr. H. Irle und Hr. Dr. R. Wirtmüller für die kritische Durchsicht des Manuskripts und wertvolle Hinweise. 


\section{Zusammenfassung}

Aufgabe der Sozialmedizin ist, Regeln zu erarbeiten, wer wann wo am besten und kostengünstigsten zu behandeln ist, inklusive der Organisation des Versorgungssystems. Im gegliederten Sozialsystem sind die Leistungsprinzipien der Versicherung, Versorgung und Fürsorge zu unterscheiden. Das Sozialgesetzbuch gliedert sich in zwölf Teile.

Circa ein Viertel von Patienten in Allgemeinarztpraxen leiden an einer psychischen Erkrankung, die zumeist chronisch verlaufen. Die Behandlung psychischer Erkrankungen findet vor allem durch Ärzte für Allgemeinmedizin statt.

Zum Gesamtbehandlungsplan gehören die somatische und psychische Diagnostik, sozialmedizinische Diagnostik, individuelles Fall-Management, supportive Therapie, Psychotherapie, Pharmakotherapie, Somatotherapie, Soziotherapie.

Zwischen Behandler und Patient besteht ein Dienstvertrag, d.h. der Patient hat keinen Anspruch auf Heilung sondern eine Behandlung nach bestem Wissen und Gewissen unter Zugrundelegung des Facharztstandards (lege artis). Der Behandler haftet dem entsprechend nur für Verfahrensfehler, jedoch nicht für das Behandlungsergebnis.

Der Behandelnde ist verpflichtet zu Koordination und Absprachen mit Mitbehandlern. Am Langzeitbehandlungsverlauf sind niedergelassene Vertragsärzte als Primärbehandler, sowie punktuell bspw. stationäre Rehabilitationskliniken und Richtlinienpsychotherapeuten beteiligt. Die Behandlung psychischer Erkrankungen findet im Sinne des ICF-Modells statt auf den Ebenen der Funktion bzw. Symptomatik (Remission einer depressiven Episode, Abbau von Vermeidungsverhalten bei Angsterkrankungen), der Fähigkeiten (Soziale Kompetenzen, Selbstmanagement, Problemlösefähigkeiten), sowie der Kontextebene im Sinne der Verbesserung der Teilhabe im sozialen und beruflichen Leben (Betriebliches Eingliederungsmanagement, stufenweise Wiedereingliederung, Leistungen zur Teilhabe).

Ärztliche Handlungen (z.B. Arbeitsunfähigkeitsfeststellung) können kontextabhängig einerseits als Begutachtungsaufgaben, andererseits auch als therapeutische Interventionen zu verstehen sein. 


\title{
Socio-medical aspects of mental disorders
}

\section{Part 2: Mental disorders in the German medical system and therapeutic interventions}

\begin{abstract}
Within the German social system one finds an amount of different institutions which are all differently connected with each other and have their own rules. The German Social Law consists of 12 parts.

About $25 \%$ of patients in general care suffer from mental disorders which in most cases take a chronic course. The treatment of mental illness is mainly done by general practitioners. Treatment consists of somatic and psychological diagnostic, socio-medical diagnostic, individual case management, psychotherapy, pharmacotherapy, somato therapy and socio therapy.

Physicians must treat a patient "lege artis" according to the present standard of medical care, however they cannot be made responsible for the outcome.

Physicians must exchange information with their cotherapists and keep themselves informed about the whole treatment process of a patient. In the long time treatment, general practitioners are primary physicians. Additional temporary treatments may take place over the course (e.g. an inpatient rehabilitation treatment, psychotherapy with a limited contingent of therapy sessions)

Treatment contains functioning (symptom reduction, e.g. remission of a depressive episode, reduction of avoidance behavior in anxiety disorders), capacity (social competences, self management, problem solving), and context (vocational reintegration, stepwise reintegration at the workplace).

Some of physicians' actions like stating sick leave are socio-medical judges on the one hand, but can also be therapeutic intervention on the other hand.
\end{abstract}




\section{Psychische Erkrankungen im medizinischen Versorgungssystem}

\subsection{Versicherungsrechtliche Zuständigkeiten}

Als Beginn des modernen deutschen Sozialsystems kann die „Kaiserliche Botschaft“ vom 17.11.1881 angesehen werden, in deren Folge sich ein differenziertes System an Sozialgesetzen entwickelt hat: das Gesetz für die Krankenversicherung der Arbeiter (1883), Unfallversicherungsgesetz (1884), Gesetz über die Invaliditäts- und Alterssicherung (1889), Angestelltenversicherungsgesetz (1911), Reichsknappschaftsgesetz Arbeitslosenversicherung (1927), Bundesversorgungsgesetz (1950), RentenversicherungsNeuregelungsgesetze (1957), Bundessozialhilfegesetz (1961), Beginn der Kodifikation des Sozialgesetzbuches (1976), Gesundheitsreformgesetz (1989), Rentenreformgesetz (1991),

Pflegeversicherungsgesetz (1995), Sozialgesetzbuch IX (2001). Heute ist das Sozialgesetzbuch in zwölf Teile untergliedert (Tab. 1).

Tabelle 1: Gliederung des Sozialgesetzbuches

\begin{tabular}{ll}
\hline SGB I & Allgemeiner Teil \\
SGB II & Grundsicherung \\
SGB III & Arbeitsförderung \\
SGB IV & Gemeinsame Vorschriften \\
SGB V & Gesetzliche Krankenversicherung \\
SGB VI & Gesetzliche Rentenversicherung \\
SGB VII & Gesetzliche Unfallversicherung \\
SGB VIII & Kinder- und Jugendliche \\
SGB IX & Rehabilitation und Teilhabe \\
SGB X & Verwaltungsverfahren \\
SGB XI & Soziale Pflegeversicherung \\
SGB XII & Sozialhilferecht \\
\hline
\end{tabular}

Zur Systematisierung des Sozialrechts unterscheidet man die Trias: Versicherung, 
Versorgung, Fürsorge (Tab. 2). Für Versicherung charakteristisch ist die Verbindung von beitragsfinanzierter Selbsthilfe und sozialem Ausgleich innerhalb der Versicherungsgemeinschaft sowie zwischen ihr und dem Staat. Ökonomisch definiert dient die Versicherung der planmäßigen Deckung eines Geldbedarfs, der im Einzelfall ungewiss, insgesamt jedoch schätzbar ist. Die Versicherung soll einen Risikoausgleich herbeiführen durch Zusammenfassung einer genügend großen Zahl von Personen, die alle von einem Risiko oder mehreren Risiken bedroht sind, ohne diese - zufälligen - Risiken gleichzeitig oder in jedem Falle zu realisieren (Gefahrengemeinschaft). In diesem Sinne der Versicherung zuzuordnen sind die gesetzliche Krankenversicherung, gesetzliche Unfallversicherung, sowie zum Teil die gesetzliche Rentenversicherung, soweit sie nicht über Staatszuschüsse, sondern aus Beiträgen gedeckt werden.

In der Versorgung besteht kein Gegenseitigkeitsverhältnis. Die Finanzierung erfolgt ausschließlich aus Steuermitteln. Versorgungsleistungen entschädigen für Opfer, die dem Staat erbracht wurden (Kriegsopfer) oder für die der Staat die - zumindest politische Verantwortung (Opfer von Gewaltverbrechen) übernommen hat. In diesem Sinne der Versorgung zuzuordnen sind Leistungen an Kriegsopfer (Militär- u. Zivilpersonen), Leistungen an Verfolgte des „3. Reiches“ nach dem Bundesentschädigungsgesetz, Entschädigung von Verbrechensopfern nach dem Gesetz über die Entschädigung für Oper von Gewalttaten (OEG) oder Entschädigung für Impfschäden (§ 60 IfSG). Zuständig sind Versorgungsämter und Landesversorgungsämter.

Die Fürsorge wird finanziert aus allgemeinen Steuermitteln im Wege gesamtgesellschaftlicher Solidarität. Fürsorgeleistungen sollen denjenigen die Führung eines menschenwürdigen Lebens ermöglichen, die aus eigener Kraft oder unter Zuhilfenahme fremder Mittel auf Dauer oder vorübergehend nicht in der Lage sind, ihren Lebensunterhalt zu finanzieren. Es gilt der Grundsatz der Subsidiarität. Art, Form und Maß der Hilfe richten sich nach der persönlichen Situation des Hilfeempfängers und nach der Art seines Bedürfnisses. In diesem Sinne der Fürsorge zuzurechnen sind Leistungen nach dem SGB XII, z.B. Arbeitslosenhilfe, Jugendhilfe, Kinder- und Wohngeld.

Tabelle 2: Auswahl an Versicherungs- und Fürsorgeinstitutionen 


\begin{tabular}{|c|c|c|}
\hline Institution & Risiken & Aufgaben \\
\hline Krankenversicherung & Krankheit, Pflegebedürftigkeit & $\begin{array}{l}\text { Krankenbehandlung, } \\
\text { Gesundheitsförderung, } \\
\text { Früherkennung, } \\
\text { Krankheitsverhütung }\end{array}$ \\
\hline Unfallversicherung & $\begin{array}{l}\text { Arbeitsunfall, Berufskrankheit, } \\
\text { Tod }\end{array}$ & $\begin{array}{l}\text { Unfallverhütung, } \\
\text { Teilhabeleistungen, Renten }\end{array}$ \\
\hline Rentenversicherung & Erwerbsminderung, Alter, Tod & Teilhableistungen, Renten \\
\hline Arbeitsförderung & $\begin{array}{l}\text { Arbeitslosigkeit, } \\
\text { Erwerbsminderung }\end{array}$ & $\begin{array}{l}\text { Berufsberatung, } \\
\text { Arbeitsvermittlung, } \\
\text { Teilhabeleistungen }\end{array}$ \\
\hline Öffentliche Jugendhilfe & $\begin{array}{l}\text { Geistige und Seelische } \\
\text { Behinderung von Kindern und } \\
\text { Jugendlichen }\end{array}$ & $\begin{array}{l}\text { Teilhabeleistungen, } \\
\text { Eingliederungshilfe }\end{array}$ \\
\hline Sozialhilfe & Hilfebedürftigkeit & $\begin{array}{ll}\text { Teilhabeleistungen, } & \text { Hilfe zum } \\
\text { Lebensunterhalt, } & \text { Hilfe in } \\
\text { besonderen } & \text { Lebenslagen } \\
\text { (Blinden-, Altenhilfe) }\end{array}$ \\
\hline $\begin{array}{l}\text { Soziales } \\
\text { Entschädigungsrecht }\end{array}$ & $\begin{array}{l}\text { Kriegsopferversorgung, } \\
\text { Hinterbliebenenversorgung, } \\
\text { Opfer von Gewalttaten, } \\
\text { Impfgeschädigte, politische } \\
\text { Häftlinge (ehem. DDR) }\end{array}$ & $\begin{array}{l}\text { Renten, Übernahme von } \\
\text { Behandlungskosten, } \\
\text { Sachleistungen (Behandlungen) }\end{array}$ \\
\hline
\end{tabular}

\subsection{Versorgungsepidemiologie psychischer Erkrankungen}

Ein Arbeitsfeld der Sozialmedizin ist die Beschreibung, Bewertung und Konzeptentwicklung des medizinischen Versorgungssystems. Eine grundlegende Methodik hierfür ist die Versorgungsepidemiologie, d.h. wer wird warum von wem wann behandelt.

Im Jahr 2008 waren von 120472 Vertragsärzten in Deutschland (Bundesarztregister der KBV 2008) 40862 Allgemeinmediziner, 4897 Nervenärzte und 4747 ärztliche Psychotherapeuten. 
Die Zahl der Allgemeinärzte wie auch der Nervenärzte/Neurologen/Psychiater blieb dabei im Zeitraum von 2004-2008 relativ stabil, während die Zahl der ärztlichen Psychotherapeuten jährliche Zuwachsraten von $3-10 \%$ aufwies. Hinzu kommen Psychologische Psychotherapeuten. Hinsichtlich der Psychotherapieverfahren sind über $80 \%$ der ärztlichen Psychotherapeuten primär tiefenpsychologisch oder analytisch orientiert, gegenüber $12 \%$ mit verhaltenstherapeutischer Orientierung. Von den Psychologischen Psychotherapeuten arbeiten derzeit $47 \%$ verhaltenstherapeutisch und 53\% tiefenpsychologisch und/oder analytisch.

Die Behandlung psychischer Erkrankungen findet vor allem durch Ärzte für Allgemeinmedizin statt (Dilling et al. 1984; Maier et al 1996; Wittchen \& Jacobi 2001). 25$30 \%$ aus Stichproben in Allgemeinarztpraxen befragter Patienten litten zum jeweiligen Untersuchungszeitpunkt an einer psychischen Erkrankung (Maier et al, 1996). Es muss daher gerade auch im allgemein- und hausärztlichen Sektor eine gute Kompetenz zur Diagnostik und Therapie psychischer Störungen vorhanden sein. Hierfür gibt es seit 1992 die psychosomatische Grundversorgung, die eine curriculare Weiterbildung voraussetzt und die seit 2008 zwingender Teil der Weiterbildung zum Arzt für Allgemeinmedizin, Innere Medizin und Gynäkologie ist. Ziele sind die Basisdiagnostik, Früherkennung und Therapie von psychischen Störungen im primärärztlichen Bereich.

Es gibt ca. 60.000 Ärzte mit einem entsprechenden Weiterbildungsabschluss. 94\% aller Nervenärzte, $68 \%$ aller Frauenärzte, $62 \%$ aller Allgemein- oder praktische Ärzte nehmen teil an der psychosomatischen Grundversorgung. Von allen in den Bereichen Neurologie, Psychiatrie, Psychosomatik und Psychotherapie abgerechneten Leistungen fielen etwa 22\% auf die psychosomatische Grundversorgung. Davon wurden 41\% von Hausärzten, 37\% von Frauenärzten, jeweils 2-3\% von Urologen, Nervenärzten, Hautärzten oder Orthopäden erbracht (Kassenärztliche Bundesvereinigung, Bundesarztregister 2001).

Im ambulanten Bereich kommen als weitere Träger der Versorgung Institutsambulanzen psychiatrischer Kliniken, Psychiatrische oder Psychosomatischen Polikliniken an Universitätskliniken, Institutsambulanzen an psychologischen Universitätsinstituten, und Ambulanzen privater Ausbildungsinstitute hinzu. Des Weiteren nahmen 2006 nach Angaben der Deutschen Arbeitsgemeinschaft für Jugend- und Eheberatung e.V. 12.154 psychosoziale Beratungsstellen an der Versorgung psychisch Kranker teil mit etwa 9550 psychotherapeutisch 
tätigen Mitarbeitern. Beratungsschwerpunkte sind vielfältig: Ehe-, Familien- und Lebensberatung, Erziehungsberatung, Krisenintervention, Frauenberatung, Beratung Alleinerziehender, Suchtberatung, Beratung für psychisch Kranke, Ausländerberatung, Sexualberatung, Familienplanungsberatung, Beratung bei chronischen Krankheiten (Dahlbender 2000; RKI 2008).

Im Bundesgesundheitssurvey (Wittchen \& Jacobi, 2001) wurde gefunden, dass 36\% der Menschen mit psychischen Störungen mindestens einmal im Leben eine Behandlung wegen ihrer psychischen Erkrankung in Anspruch nahmen, davon 51\% in zwei oder mehr Behandlungseinrichtungen. 42\% der Behandelten besuchten den Hausarzt, davon ein Drittel ausschließlich. Des Weiteren besuchten 34\% der wegen psychischer Erkrankungen behandelten Menschen Beratungsstellen, 32\% einen Nervenarzt, 27\% einen Psychologischen Psychotherapeuten, $17 \%$ einen ärztlichen Psychotherapeuten, 8\% psychotherapeutische Ambulanzen, und 23\% nahmen stationäre Psychotherapie in Anspruch. Pro Jahr bleiben allerdings auch zwei Drittel aller psychischen Störungen unbehandelt (Wittchen, 2005), wobei nicht gesagt werden kann, ob und ggf. welche Behandlungsindikation gegeben ist. Wenn eine psychische Störung erkannt wird, wird am häufigsten mit Psychopharmaka behandelt, Psychotherapie wird nur selten als alleinige Behandlung angeboten. Unterbehandlung ist besonders häufig bei Kindern und jungen Erwachsenen, bei Angststörungen und Substanzstörungen zu beobachten.

\subsection{Behandlungskoordinierung in der Versorgung psychischer Erkrankungen}

Eine Aufgabe der Sozialmedizin ist, Regeln zu erarbeiten, wer wann wo am besten und kostengünstigsten zu behandeln ist, inklusive der Organisation des Versorgungssystems. Der Patient hat einen Anspruch auf Behandlung nach dem jeweils aktuellen Facharztstandard. Es gilt das Prinzip der Behandlung nach Notwendigkeit, Zweckmäßigkeit und Wirtschaftlichkeit. Zum Gesamtbehandlungsplan gehören die somatische Diagnostik, psychische Diagnostik, sozialmedizinische Diagnostik, individuelles Fall-Management, supportive Therapie, Psychotherapie, Pharmakotherapie, Somatotherapie, Soziotherapie. Zugrunde gelegt wird in der Regel ein mehrdimensionales Krankheits- und Behandlungskonzept (Tab. 3) mit der biologischen Dimension (z.B. Pharmakotherapie, Schlafentzug, somatische Behandlung), der 
sozialen Dimension (z.B. Soziotherapie, Sozialmedizin) sowie der psychologischen Dimension (z.B. Patientenführung, Psychotherapie). Zwischen Behandler und Patient besteht ein Dienstvertrag, d.h. der Patient hat keinen Anspruch auf Heilung sondern eine Behandlung nach bestem Wissen und Gewissen unter Zugrundelegung des Facharztstandards (lege artis). Der Behandler haftet dem entsprechend nur für Verfahrensfehler, nicht jedoch das Behandlungsergebnis.

Tabelle 3: Mehrdimensionale Behandlung

\begin{tabular}{ll}
\hline mehroptional & mehrere Lösungen für ein Problem \\
\hline mehrinterventionell & Mehrere Interventionen zur Lösung eines Problems \\
\hline mehrstufig & Mehrere Schritte zur Lösung eines Problems \\
\hline mehrsyndromal & Mehrere Therapieziele zur Lösung eines Problems \\
\hline mehraxial & Mehrere Interventionen zur Lösung verschiedener Probleme \\
\hline mehraktional & Mehrere Therapeuten zur Lösung eines oder mehrerer Probleme \\
\hline
\end{tabular}

Die Koordinierung der Behandlung beinhaltet eine Abstimmung im Solidarsystem mit Blick auf den Empfänger von Sozialleistungen (Für wen sollte etwas getan werden?), die Erbringer (Welcher Therapeut wird benötigt und trägt zu einem guten Ergebnis bei?), die Art (Welche Art von Intervention ist nötig und zweckmäßig?) und den Zeitpunkten (Wann sollte wer was tun?). Wie bedeutsam eine gute Behandlungskoordinierung ist belegt, dass bei 23\% der Fälle bei der Kommission für ärztliche Behandlungsfehler bei der Ärztekammer Nordrhein ein Koordinationsverschulden festgestellt wurde, davon in 65\% ohne individuelle fachliche Fehler (Hansis 2001).

Die Behandlungskoordinierung ist auch ein wesentlicher Aspekt bei Planungen im Gesundheitswesen, d.h. Regelungen unter welchen Bedingungen ein Patient welche Hilfsangebote nutzen kann, welche Ärzte er nacheinander oder gleichzeitig kontaktieren kann, wann er in ein Krankenhaus oder eine Rehabilitationsklinik eingewiesen werden sollte, wie Doppeluntersuchungen vermieden werden können, wie Maßnahmen eines Behandlers von einem anderen fortgeführt werden können, welche Angebote unter Kosten- und Qualitätsgesichtspunkten miteinander konkurrieren u.v.m. Auf diesem Hintergrund sind 
Diskussionen um die Praxisgebühr, die Einführung der Gesundheitskarte, die Rolle der Hausärzte, um Case Manager der Krankenkassen, um die Erlaubnis von Krankenhäusern zur Erbringung ambulanter Leistungen, um Psychiatrische Institutsambulanzen usw. zu verstehen. Idealerweise sollten solche Strukturdebatten und -entscheidungen unter Hinzuziehung sozialmedizinischer Kompetenz erfolgen.

\subsection{Aufklärungs-, Dokumentations-, Auskunfts- und Schweigepflicht aus sozialrechtlicher Sicht}

Grundsätzlich gilt, dass Behandler eines Patienten sich gegenseitig zu informieren haben. Hierfür kann von einem generellen Einverständnis des Patienten ausgegangen werden. In der (Muster)berufsordnung der Bundesärztekammer heißt es in $\S 9$ Abs. 4 zur Schweigepflicht

„Wenn mehrere Ärztinnen und Ärzte gleichzeitig oder nacheinander dieselbe Patientin oder denselben Patienten untersuchen oder behandeln, so sind sie untereinander von der Schweigepflicht insoweit befreit, als das Einverständnis der Patientin oder des Patienten vorliegt oder anzunehmen ist.“

Lehnt ein Patient ab, dass Behandler sich gegenseitig informieren, würde dies in der Regel einen Behandlungsabbruch erzwingen, da eine Behandlung ohne fachgerechte Information ein Kunstfehler wäre.

Für den Austausch von Patientenunterlagen unter ärztlichen Kollegen heißt es gemäß $§ 73$ Abs. 1b SGB V ,die behandelnden Leistungserbringer sind berechtigt, mit schriftlicher Einwilligung des Versicherten, die widerrufen werden kann, die für die Behandlung erforderlichen Behandlungsdaten und Befunde bei dem Hausarzt und anderen Leistungserbringern zu erheben und für die Zwecke der von ihnen zu erbringenden Leistungen zu verarbeiten und zu nutzen.“

Patienten haben einen Anspruch darauf, dass ein Arzt nichts an Dritte mitteilt, was er im Rahmen seiner beruflichen Tätigkeit erfahren hat. Verletzungen der Schweigepflicht sind nach $\S 203$ StGB (Verletzung von Privatgeheimnissen) ein Straftatbestand. Es gilt nur eine Offenbarungsbefugnis bei rechtfertigendem Notstand ( $\$ 34 \mathrm{StGB}$ ).

Diese Absolutheit der Schweigepflicht gilt im Sozialrecht nicht. Hier greifen gemäß $§ 276$ 
SGB V andere Regeln als bei der sonstigen ärztlichen Schweigepflicht. "Haben die Krankenkassen nach $\S 275$ Abs. 1 bis 3 eine gutachtliche Stellungnahme oder Prüfung durch den Medizinischen Dienst veranlasst, sind die Leistungserbringer verpflichtet, Sozialdaten auf Anforderung des Medizinischen Dienstes unmittelbar an diesen zu übermitteln, soweit dies für die gutachtliche Stellungnahme und Prüfung erforderlich ist“. Was „erforderlich“ ist, ist im Einzelfall zu bestimmen und kann die gesamte Krankenakte umfassen, gegebenenfalls aber auch nur einzelne Informationen.

Es gibt ein Recht des Patienten auf Selbstbestimmung und Einwilligung in jegliche ärztliche Maßnahme. Die Voraussetzung dafür ist eine Aufklärung durch den Arzt. Daraus folgt auch eine uneingeschränkte Akteneinsicht für den Patienten. Das schützenswerte Interesse des Patienten an ihn betreffenden Daten überwiegt auch die Persönlichkeitsrechte des Therapeuten. Eine Unterteilung in einen objektiven und subjektiven Teil der Krankengeschichte hat in der Regel keinen Bestand. Dennoch sind bei der Aushändigung von Krankenunterlagen an den Patienten andere Rechtsnormen zu bedenken, wie beispielsweise nach $\S 19$ Abs. 4 Nr. 3 BDSG der Schutz von Interessen Dritter, z.B. fremdanamnestische Daten.

Die Dokumentationspflicht ist eine Nebenpflicht zur Behandlung im Rahmen des Behandlungsvertrags weil von Bedeutung für die Fortsetzung der Behandlung durch Andere,

für Rentenanträge, Klärung von Haftpflichtfragen. Bei Unterlassung ergeben sich Schadenersatzansprüche gegenüber dem Therapeuten. Dies ist insbesondere relevant bzgl. der Dokumentation über Patientenaufklärung oder bei der Frage von Behandlungsfehlern.

\subsection{Vergütung ambulanter Behandlungen}

Grundlage des Einheitlichen Bewertungsmaßstabes (EBM), dem Vergütungssystem der ambulanten Versorgung, ist das SGB V. In $\S 87$ Abs. 2 SGB V ist festgelegt: "Der einheitliche Bewertungsmaßstab bestimmt den Inhalt der abrechnungsfähigen Leistungen und ihr wertmäßiges, in Punkten ausgedrücktes Verhältnis zueinander; soweit möglich, sind die Leistungen mit Angaben für den zur Leistungserbringung erforderlichen Zeitaufwand des Vertragsarztes zu versehen". Der EBM gilt für die ambulante Behandlung von Patienten der 
Gesetzlichen Krankenversicherung. (Bei Privatpatienten gilt analog die Gebührenordnung für Ärzte GOÄ).

Die Abrechnung erfolgt mit der zuständigen Kassenärztlichen Vereinigung, wenn der Arzt als Kassenarzt zugelassen oder als Krankenhausarzt ermächtigt ist oder - wenn er beides nicht ist in der Notfallbehandlung. Eine „Ermächtigung“ von Krankenhausärzten kommt nur in Betracht, wenn im Angebot der niedergelassenen Vertragsärzte eine Versorgungslücke besteht.

Der EBM ist in sechs Teile gegliedert: Arztgruppenübergreifende allgemeine Leistungen, Allgemeine diagnostische und therapeutische Leistungen, Arztgruppenspezifische Leistungen, Arztgruppenübergreifende spezielle Leistungen, Kostenpauschalen, Anhänge. Mit der Gesundheitsreform 2007 hat der Gesetzgeber den Bewertungsausschuss verpflichtet einen neuen EBM zu beschließen, in dem insbesondere vermehrt Leistungen der Hausärzte, teilweise auch der Fachärzte, zu Pauschalen zusammengefasst werden. Die aktuelle Version des EBM sowie die Übersicht der Änderungen (2009) ist bei der Kassenärztlichen Bundesvereinigung einsehbar (http://www.kbv.de/ebm2010/ebmgesamt.htm).

\section{Spezielle therapeutische Maßnahmen in der Sozialmedizin}

Während epidemiologische Fragestellungen und Untersuchungen, oder die Gestaltung des Versorgungssystems strukturelle Aspekte des Gesundheitswesens zum Gegenstand haben, gibt es auch eine Reihe sozialmedizinischer therapeutischer Interventionen, die den einzelnen Patienten betreffen. Diese sollen im Folgenden kurz dargestellt werden.

\subsection{Arbeitsunfähigkeit und Erwerbsunfähigkeit als therapeutische Maßnahme}

Eine Arbeitsunfähigkeit wird festgestellt, wenn erkrankungsbedingt die aktuelle berufliche Tätigkeit nicht oder nur unter Verschlimmerung der Erkrankung ausgeübt werden kann (Arbeitsunfähigkeits-Richtlinie GBA 2004). Da das Behandlungsziel die Verbesserung des Erkrankungszustands bzw. die Linderung von Leiden ist, kann eine vorübergehende oder dauerhafte Herausnahme eines Patienten aus seinem aktuellen Arbeitskontext für eine 
Entlastung sorgen und bereits eine Befindensverbesserung zur Folge haben, und die Grundlage für eine störungsspezifische Therapie verbessern.

Insofern dient die Arbeitsunfähigkeitsfeststellung auch als therapeutische Maßnahme.

Sinnvoll kann eine Arbeitsunfähigkeitsbescheinigung bspw. sein zur Stabilisierung des Biorhythmus bei einer Patientin mit einer depressiven Erkrankung die in wechselnden Schichtdiensten arbeitet, oder zur Reduktion von Reizüberflutung bei einem Patienten mit psychotischer Symptomatik der in einer Zeitungsredaktion ständig mit neuen visuellen und auditiven Informationen konfrontiert ist.

Erwerbsunfähigkeitsfeststellung als therapeutische Maßnahme kann als dauerhafte Entlastung solcher Patienten dienen, bei denen die spezifischen oder auch generelle berufliche Anforderungen oder der Kontext nicht nur vorübergehend während einer Erkrankungsepisode, sondern dauerhaft starke Risikofaktoren für Rezidive und Krankheitsverschlechterung darstellen.

Bei spezifischen arbeitsplatzbezogenen Angsterkrankungen (Linden \& Muschalla 2007), bei denen sich speziell bezüglich des Arbeitsplatzes ein Vermeidungsverhalten manifestiert, ist bei der Frage der Arbeits(un)fähigkeit stets zu berücksichtigen, dass hier eine längerdauernde Arbeitsunfähigkeit die arbeitsbezogenen Ängste sogar verstärken kann.

Eine Herausnahme aus der beruflichen Tätigkeit bzw. aus der Erwerbsarbeit ist also eine therapeutische Maßnahme, die in den Gesamtbehandlungsprozess eingepasst werden und in jedem Fall von weiteren erkrankungsspezifischen therapeutischen Maßnahmen flankiert werden muss.

\subsection{Unterbringung psychisch Kranker}

Wenn ein Patient aufgrund einer psychischen Erkrankung und einer damit einhergehenden Selbst- oder Fremdgefährdung geschützt werden muss, dann kann bei fehlender Einsicht eine Zwangseinweisung in eine psychiatrische Klinik erfolgen.

Gesetze zur Unterbringung psychisch Kranker (PsychKG) sind Landesgesetze. Eine Grundregel ist, dass eine stationäre Hilfe nur dann unabhängig vom erklärten Willen des Betroffen geleistet werden darf, wenn das Ziel der Hilfe nicht auf anderem Weg erreicht werden kann. Die Unterbringung psychisch Kranker gegen oder ohne den eigenen Willen 
erfolgt nur wenn und solange durch krankheitsbedingtes Verhalten ihr Leben, ernsthaft ihre Gesundheit oder besonders bedeutende Rechtsgüter Anderer gefährdet sind und Gefährdungen nicht anders abgewendet werden können. Auf Antrag des Gesundheitsamts (ggfls. Bezirksamt, Landratsamt) erfolgt eine gerichtliche Entscheidung über die stationäre Behandlung nach Anhörung eines Sachverständigen, Anhörung von Eltern oder Kindern, Anhörung des Betreuers, Anhörung einer Person des eigenen Vertrauens, des Leiters der Einrichtung, in dem der Betroffene lebt oder behandelt werden soll und unter Beiordnung eines Verfahrenspflegers/Rechtsanwalts. In Notfällen erfolgt eine vorläufige Unterbringung bis zum Ablauf des nächsten Tages durch das Gesundheitsamt / den Sozialpsychiatrischen Dienst unter Hinzuziehung der Polizei. Besondere Schutzmaßnahmen sind möglich (z.B. Fixierung, Wegnahme von Gegenständen). Der nach PsychKG Untergebrachte hat zwar die Unterbringung bzw. befristete Fixierung zu dulden, aber keine über die Notfallsituation hinaus reichenden pharmakologischen Behandlungsmaßnahmen, das heißt, nur solange z.B. noch unmittelbare massive Fremdgefährdung besteht.

\subsection{Geschäftsfähigkeit und Betreuung psychisch Kranker}

Erwachsene, die aufgrund einer psychischen Krankheit oder einer körperlichen, geistigen oder seelischen Behinderung ihre Angelegenheiten ganz oder teilweise nicht besorgen können, erhalten Hilfen über das Betreuungsgesetz (BtG). Eine Betreuung ist nicht mit Geschäftsunfähigkeit ( $§ 104$ BGB) gleichzusetzen. Das Betreuungsgericht bestellt auf seinen Antrag oder von Amts wegen einen oder mehrere Betreuer. Festzulegen sind Gegenstand, Umfang und Dauer der Betreuung. Durch das Betreuungsgericht genehmigungspflichtig sind z.B. Unterbringung, Wohnungsauflösung oder eingreifende Behandlungen. Geldgeschäfte sind nur bei Anordnung eines Einwilligungsvorbehaltes genehmigungspflichtig.

\subsection{Der öffentliche Gesundheitsdienst und die Sozialpsychiatrischen Dienste}

Eine wesentliche Aufgabe der Sozialmedizin ist die Primärprophylaxe von Krankheiten. Im Bereich der psychischen Störungen gehören dazu Beratungsangebote zur Unterstützung bei der Bewältigung kritischer Lebenssituationen. Dabei bezieht sich die institutionelle Beratung auf „Menschen, die in Fragen der allgemeinen Lebensplanung, der Gestaltung von 
menschlichen Beziehungen und im Umgang mit Konflikten und Entwicklungsproblemen in Partnerschaft, Ehe und Familie nach Veränderungen und neuen Lösungen suchen. Beratung hat prozesshaften Charakter und ist darauf angelegt, dass Ratsuchende mit ihren Fragen und Problemen besser umgehen können und eigene Lösungswege erarbeiten.“ (DAJEB 2002).

In Beratungsstellen des öffentlichen Gesundheitsdienstes wie auch von Sozialverbänden wird keine Krankheitsbehandlung durchgeführt, sondern, wie bereits ausgeführt, Unterstützung in kritischen Lebenssituationen gegeben, von Schwangerschaft, über Familienkonflikte, soziale Notlagen oder auch Suchtprobleme. Die Finanzierung von Beratungsstellen erfolgt sehr unterschiedlich, z.B. über Pflichtleistungen (Erziehungsberatungsstellen) oder Zuschüsse der Kommunen oder häufig auch Eigenmittel des Trägers. Sie können von den Ratsuchenden in der Regel kostenfrei in Anspruch genommen werden.

Der sozialpsychiatrische Dienst ist Teil des öffentlichen Gesundheitsdienstes und erfüllt Pflichtaufgaben der Kreise und kreisfreien Städte. Sozialpsychiatrische Dienste sind i. d. R. den Gesundheitsämtern angegliedert. In den Empfehlungen der Expertenkommission der Bundesregierung zur Reform der Versorgung im psychiatrischen, psychotherapeutischpsychosomatischen Bereich vom November 1988 sind für Sozialpsychiatrische Dienste bestimmte Aufgaben und Arbeitsweisen formuliert: Beratung, vorsorgende und nachgehende Hilfen, ärztlich geleitete Sprechstunden, Hausbesuche, Koordination der Einzelhilfen. Die Integration seelisch kranker Menschen in die Gemeinschaft ist für alle sozialpsychiatrischen Dienste ein wesentliches Ziel. Die Zusammenarbeit mit anderen Institutionen der Region im Rahmen eines gemeindepsychiatrischen Ansatzes ist ein wichtiges Merkmal der Arbeit. Aus der umfassenden Kenntnis der jeweiligen regionalen Versorgungslandschaft ergeben sich darüber hinaus Koordinationsaufgaben.

\subsection{Vertragsärzte}

Psychische Erkrankungen werden in der Mehrheit der Fälle von Ärzten für Allgemeinmedizin behandelt (Linden 2001). Dies ist alleine schon aus quantitativen Gründen unumgänglich, da nicht vorstellbar ist, dass ca. 31\% der Bevölkerung, die aktuell unter psychischen Störungen 
leiden, fachärztlich oder fachpsychotherapeutisch zu betreuen wären. Dementsprechend fand sich bei den Erhebungen des Bundesgesundheitssurveys (Wittchen et al. 1999; Wittchen \& Jacobi 2001), dass z.B. depressive Personen im Mittel während des vergangenen Jahres 14,5 Kontakte zu Hausärzten, aber nur 1,4 zu Nervenärzten/Psychiatern hatten. Dieses Verhältnis von 1:10 entspricht in etwa dem Verhältnis von 7'916 niedergelassenen psychiatrischen und psychosomatischen Fachärzten zu 60'529 niedergelassenen Allgemeinärzten und Internisten. Es gibt umfangreiche Untersuchungen zu psychischen Erkrankungen in der hausärztlichen Praxis (Wittchen 2000; Linden et al 1996, 2000; Dittmann et al 1997). Hausärzte behandeln Ersterkrankungen und leichtere Störungen, aber auch rezidivierende und lebenslange

Erkrankungen. Sie sind vor allem auch Case-Manager wenn Spezialisten oder stationäre Akutoder Reha-Behandlungen keine Remission erbringen könnten.

Die Aufgabe von „Fachärzten für Nervenheilkunde“, „Fachärzten für Psychiatrie“, „Fachärzten für Psychiatrie und Psychotherapie“ oder „Fachärzten für Psychosomatik und Psychotherapie“ ist vor allem die fachärztliche Behandlung von Patienten mit therapierefraktären oder chronischen psychischen Erkrankungen.

Die Betreuung chronisch Kranker durch niedergelassene Vertragsärzte ist auf Jahre und Jahrzehnte angelegt. Daraus ergeben sich spezifische rehabilitations- und sozialmedizinische Arbeitsaufträge (Tab. 4).

Tabelle 4: Diagnostik, Behandlung und Begutachtungsaufgaben niedergelassener Vertragsärzte

\begin{tabular}{lll}
\hline Diagnostik & - & frühzeitiges Erkennen eines beginnenden Chronifizierungsprozesses \\
& - & Abklärung von medizinischen Faktoren, die eine Chronifizierung \\
& begünstigen \\
& - Abklärung von Kontextfaktoren, die eine Chronifizierung begünstigen \\
& - & frühzeitiges Erkennen sich abzeichnender beruflicher oder sozialer \\
& Teilhabestörungen \\
\hline Behandlung & - & mehrdimensionale Therapie \\
& - & chronifizierende Erkrankungen sind per definitionem therapieresistent, \\
\hline
\end{tabular}


dies erfordert ein spezielles und intensiviertes Behandlungsvorgehen.

- Behandlungszeiträume: Man muss man in der Regel Monate, wenn nicht Jahre warten, bevor festzustellen ist, ob eine bestimmte Behandlung wirksam ist.

- Patientenführung und Compliance: Patienten müssen sehr sorgfältig über die Art der geplanten Therapie informiert werden.

- Dokumentation des Erkrankungszustandes muss über längere Zeit durchgeführt werden.

- Therapieziel ist nicht unbedingt eine remissio ad integrum, sondern oft nur eine palliativ orientierte Reduktion der Krankheitsschwere oder der Rezidivfrequenz.

- Partizipationsorientierte Pharmakotherapie

- psychoedukative und psychotherapeutische Behandlungsansätze: Komorbidität (z.B. Persönlichkeitsakzentuierungen), Krankheitsbewältigung, funktionale Lebensstilanpassung, „,salutotherapeutische“ Maßnahmen zum Erhalt gesunder Lebensbereiche

\begin{tabular}{ll}
\hline Sozial- & - Krankheitssymptomen einerseits und Kapazitäts- und \\
medizinische & Partizipationsstörungen unterscheiden \\
Begutachtung & - Bei gegebener Symptomatik (Funktionsstörung) ist nicht nur zu prüfen, \\
& welche „Kapazitätsstörungen“ daraus erwachsen sondern auch welche \\
& Möglichkeiten zur Anpassung des Umfeldes (z.B. des Arbeitsplatzes) \\
& bestehen, um trotz Kapazitätsstörungen eine Partizipationsstörung \\
& vermeiden zu können \\
- & Feststellung einer Arbeits- und ggf. Erwerbsfähigkeit \\
- & „Anwalt“ des Patienten \\
- & Beurteilung von Arbeitsplatzanforderungen im Bezug auf gesundheitliche \\
& Einschränkungen
\end{tabular}

\begin{tabular}{lll}
\hline Sozio- & - & Kenntnisse sozialer und beruflicher Reintegrationsmöglichkeiten \\
therapeutische, & - & Beraten bezüglich notwendiger Arbeitsplatzveränderungen und Auskunft \\
Sozial- & welche Charakteristika ein leidensgerechter Arbeitsplatz haben muss
\end{tabular}




\begin{tabular}{lll}
\hline medizinische, & - Einleitung von Maßnahmen zur medizinischen beruflich-orientierten \\
rehabilitative & Rehabilitation \\
Interventionen & - im Bedarfsfall eine gestufte Wiedereingliederung einleiten \\
& - entscheiden, welche Patienten wann in eine Rehabilitationsklinik kommen \\
- & Nach Ende einer stationären Rehabilitationsmaßnahme eingeleitete \\
& Maßnahmen ambulant fortführen \\
- & „Lotsen“ im Gesundheitssystem
\end{tabular}

\subsection{Richtlinienpsychotherapie}

Eine Richtlinienpsychotherapie ist keine Akutbehandlung, sondern inhaltlich, strukturell, rechtlich und ethisch in der Mehrzahl der Fälle eine rehabilitative Maßnahme und daher zumindest partiell als sozialmedizinische Intervention zu verstehen.

Richtlinienpsychotherapie ist eine beantragte, begutachtete und bewilligte, zeitlich begrenzte Behandlungsform für psychische Erkrankungen, die in den Psychotherapierichtlinien (1998) geregelt ist. Sie steht nicht für Fragen der allgemeinen Lebensberatung zur Verfügung. Nach maximal fünf probatorischen Sitzungen muss vom Therapeuten ein Bericht für einen Gutachter (ggf. ergänzt durch einen ärztlichen Konsiliarbericht, Vorbefunde und Klinikberichte) erstellt werden und die Therapie vom Patienten bei der Krankenkasse beantragt werden. Je nach Verfahren, d.h. abhängig von der Qualifikation des Psychotherapeuten, wird eine tiefenpsychologisch fundierte, eine psychoanalytische Psychotherapie, oder eine Verhaltenstherapie beantragt. Die Bewilligung erfolgt abhängig davon, ob die beantragte Behandlung ausreichend, notwendig, zweckmäßig und wirtschaftlich erscheint, und ob sich prognostisch eine Veränderung im erwünschten Sinne erwarten lässt. Es wird von Beginn an ein begrenztes Stundenkontingent festgelegt.

Da psychische Erkrankungen oft chronisch verlaufen geht es in der Regel nicht um die Vollremission einer Akuterkrankung, sondern um die Erarbeitung von Bewältigungsstrategien, Verhaltensänderungen, Verbesserung oder Kompensation im Fähigkeitsniveau, oder auch kontextbezogene Maßnahmen. Ist das bewilligte Stundenkontingent erschöpft, ist die Therapie 
zu beenden, auch dann wenn der Patient nicht ,geheilt"“ ist, sondern die Erkrankung weiter besteht. Bei der Richtlinienpsychotherapie handelt es sich also um eine „kurmäßige“ Anwendung mit von Beginn an begrenztem Umfang. Die Kontingentierung und Befristung einer Therapiemaßnahme unabhängig vom aktuellen Krankheitszustand ist bei Akuterkrankungen (Weig 2006) juristisch nicht möglich, sondern fachlich, juristisch und ethisch nur denkbar bei chronischen Erkrankungen bei denen es keine „unterlassene Hilfeleistung" gibt. Therapieziele sind dementsprechend in Anlehnung an die ICF (WHO 2001) nicht nur die Besserung von Funktionsstörungen (Psychopathologie), sondern auch von Fähigkeitsstörungen, oder der sozialen und beruflichen Partizipation. Psychotherapeuten müssen sich bewusst sein, dass sie in einem begrenzten Zeitfenster innerhalb eines sehr viel längeren Gesamtkrankheitsverlaufs intervenieren, und dass daher auch eine Koordinierung und Abstimmung mit den Vor- und Nachbehandlern von besonderer Relevanz ist. Die Richtlinienpsychotherapie hat formal, im Sinne einer Fallmanagement-orientierten Kurzzeitintervention, bei der Behandlung psychischer Erkrankungen einen ähnlichen Status wie psychosomatische Rehabilitationskliniken.

\subsection{Sozialmedizinische und therapeutische Funktion von Rehabilitationskliniken}

Rehabilitation als Leistung des Sozial- und Gesundheitssystems kommt für Patienten infrage die behindert bzw. chronisch krank sind oder von Behinderung und chronischer Erkrankung bedroht sind ( $\$ 2$ SGB IX). Neben der beruflichen Rehabilitation, unterhaltsichernden Leistungen, Leistungen zur Teilhabe am Leben in der Gemeinschaft oder Vorsorgeleistungen ist die „medizinische Rehabilitation“ eine eigenständige Form der Rehabilitation. Zu ihren Aufgaben gehört nach $\S 26$ SGB IX chronische Krankheiten abzuwenden, zu beseitigen, zu mindern, auszugleichen, eine Verschlimmerung zu verhüten oder Einschränkungen der Erwerbsfähigkeit und Pflegebedürftigkeit zu verhüten unter Anwendung von der Behandlung durch Ärzte und andere Heilberufe, Arznei- und Verbandmittel, Heilmittel, Psychotherapie usw. Analog zu beruflichen Rehamaßnahmen wird auch medizinische Rehabilitation zu einem wesentlichen Teil in stationärer oder teilstationärer (ganztags ambulanter) Form erbracht (§ 19 Abs. 2 SGB IX), d.h. in „Rehakliniken“ (Linden 2005, 2009).

Patienten werden in ,psychosomatische“ Rehabilitationskliniken eingewiesen, zur Behandlung 
wie Prophylaxe unterschiedlichster psychischer Erkrankungen (Tab. 5). Ein wichtiger Einweisungsgrund ist auch die Wiederherstellung oder der Erhalt der Arbeitsfähigkeit und die Beurteilung des Leistungsvermögens. Es kommen alle psychischen Erkrankungen zur Behandlung und nicht nur ,psychosomatische Störungen“ im engeren Sinne (Hiller et al 2007; Rehabilitationszentrum Seehof 2009).

Tabelle 5: Aufgaben der stationären medizinischen Rehabilitation

\begin{tabular}{ll}
\hline Abwehr von & Diagnostische Klärung \\
Pseudochronizität & Optimierung von Behandlungen \\
& Intensivierung von Behandlung \\
& Koordinierung von Behandlung \\
\hline Unterstützung bei der & Edukation \\
Krankheits- & Symptommanagement \\
bewältigung & Palliativtherapie \\
& Gesundheitsförderung (Salutotherapie) \\
\hline Prävention einer & Einleitung und Optimierung einer Rezidivprophylaxe \\
Verschlechterung & Therapie bzgl. Krankheitsentwicklungsfaktoren \\
\hline Prävention negativer & Arbeitsplatzinterventionen \\
Krankheitsfolgen & soziales Netz \\
\hline Sozialmedizinische & Krankheitsfeststellung \\
Leistungsbeurteilung & Beurteilung von Funktionsstörungen und Arbeitsfähigkeit \\
& Positives und negatives Leistungsbild \\
& Prognosebeurteilung \\
\hline
\end{tabular}

Die besondere Rolle der Rehabilitationsklinken als Einrichtungen des sozialmedizinischen Begutachtung ergibt sich aus dem „Arbeitsunfähigkeitsmanagement“ (vgl. Teil 1, Abschnitt 4.4) und dem Grundsatz „Reha vor Rente“. Beim AU-Management werden, wie bereits dargestellt, Patienten von der Krankenkasse aufgefordert, einen Antrag auf Einleitung einer Rehamaßnahme zu stellen, wenn eine längere oder wiederholte Arbeitsunfähigkeit absehbar ist ( $\$ 51$ SGB V). Gleichermaßen werden Patienten von den Arbeitsagenturen, Unfallkassen oder Rentenversicherungen aufgefordert, sich einer Rehamaßnahme zu unterziehen, wenn eine 
Erwerbsunfähigkeit droht oder bereits eine Rente beantragt wurde. In den Rehakliniken ist abzuklären, was das Problem ist das zur Teilhabeeinschränkung führt, und dann eine Behandlung einzuleiten. Da der bisherige Verlauf und die Therapie, und vor allem die Krankheitsentwicklung unter Therapie, die entscheidenden Kriterien für eine Prognose des weiteren Status und Verlaufs sind, ist die erweiterte Diagnostik, Zustandsbeurteilung und Therapieansprechbarkeit im Rahmen der stationären medizinischen Rehabilitation eine wichtige Grundlage für eine vertiefte sozialmedizinische Begutachtung. Unter stationären Bedingungen kann in einigen Fällen die Arbeitsfähigkeit gesichert oder wiederhergestellt werden, in anderen Fällen ist das Rehaergebnis die verlässliche Mitteilung an die Versicherungen, dass mit einer dauernden Leistungseinschränkung und damit Berentungsnotwendigkeit zu rechnen ist.

\subsection{Medizinisch-beruflich orientierte Rehabilitation (MBO) und berufliche Rehabilitation}

Soweit die Arbeitsfähigkeit Ziel der medizinischen Rehabilitation ist, spricht man von „medizinisch-beruflich orientierter Rehabilitation (MBO)““ (Hillert et al 2009; Löffler et al 2010). Die MBO umfasst medizinisch-therapeutische Maßnahmen im engeren Sinne mit unmittelbarem Arbeitsbezug. Dazu gehören neben der diagnostischen Abgrenzung von speziellen Krankheitsbildern mit unmittelbarem Arbeitsbezug (Arbeitsplatzbezogene Angsterkrankungen (Linden \& Muschalla 2007), arbeitsplatzbezogene Kränkungs- und Verbitterungsreaktionen (Muschalla \& Linden 2010a) oder arbeitsplatzbezogene Schlafstörungen und Erschöpfungssyndrome) und neben der eigentlichen Krankenbehandlung eine Reihe spezieller Fähigkeits- und Kontext- und Partizipationsorientierter Therapieoptionen und Interventionen (Hillert et al 2009). Beispiele sind ein Gruppentraining der Stressbewältigung am Arbeitsplatz (Hillert et al 2007), berufliche Belastungserprobungen (Beutel et al 1998), das Betriebliche Eingliederungsmanagement (Hetzel et al 2006; Muschalla \& Linden 2010b), oder die gestufte Wiedereingliederung.

Eine gestufte Wiedereingliederung an einem bestehenden Arbeitsplatz kann z.B. nach einer ambulanten oder stationären Rehabilitationsmaßnahme in Absprache des behandelnden Arztes mit dem Arbeitgeber des Patienten zu Lasten der Rentenversicherung (innerhalb von zwei Wochen nach einer stationären Rehabilitation) oder ansonsten der Krankenkassen 
durchgeführt werden (Tab. 6).

\section{Tabelle 6: Stufenweise Wiedereingliederung}

SGB V Können arbeitsunfähige Versicherte nach ärztlicher Feststellung

\$74 ihre bisherige Tätigkeit teilweise verrichten und können sie durch eine stufenweise Wiederaufnahme ihrer Tätigkeit voraussichtlich besser wieder in das Erwerbsleben eingegliedert werde, soll der Arzt auf der Bescheinigung über die Arbeitsunfähigkeit Art und Umfang der möglichen Tätigkeiten angeben und dabei in geeigneten Fällen die Stellungnahme des Betriebsarztes oder mit Zustimmung der Krankenkasse die Stellungnahme des Medizinischen Dienstes ( $\$ 275)$ einholen.

SGB Können arbeitsunfähige Leistungsberechtigte nach ärztlicher Feststellung

IX §28 ihre bisherige Tätigkeit teilweise verrichten und können sie durch eine stufenweise Wiederaufnahme ihrer Tätigkeit voraussichtlich besser wieder in das Erwerbsleben eingegliedert werden, sollen die medizinischen und die sie ergänzenden Leistungen entsprechend dieser Zielsetzung erbracht werden.

Betriebliches Eingliederungsmanagement (BEM, § 84 SGB IX) zielt auf die Sicherung und Erhaltung der Beschäftigungsfähigkeit von Arbeitnehmern mit gesundheitlichen Beeinträchtigungen (Tab. 7).

Tabelle 7: Betriebliches Eingliederungsmanagement

SGB Sind Beschäftigte innerhalb eines Jahres länger als sechs Wochen ununterbrochen oder

IX §84 wiederholt arbeitsunfähig, klärt der Arbeitgeber mit der zuständigen (2) Interessenvertretung im Sinne des $\S 93$, bei schwerbehinderten Menschen außerdem mit der Schwerbehindertenvertretung, mit Zustimmung und Beteiligung der betroffenen Person die Möglichkeiten, wie die Arbeitsunfähigkeit möglichst überwunden werden und mit welchen Leistungen oder Hilfen erneuter Arbeitsunfähigkeit vorgebeugt und der Arbeitsplatz erhalten werden kann (betriebliches Eingliederungsmanagement). Soweit erforderlich wird der Werks- oder 
Betriebsarzt hinzugezogen. Die betroffene Person oder ihr gesetzlicher Vertreter ist zuvor auf die Ziele des betrieblichen Eingliederungsmanagements sowie auf Art und Umfang der hierfür erhobenen und verwendeten Daten hinzuweisen. Kommen Leistungen zur Teilhabe oder begleitende Hilfen im Arbeitsleben in Betracht, werden vom Arbeitgeber die örtlichen gemeinsamen Servicestellen oder bei schwerbehinderten Beschäftigten das Integrationsamt hinzugezogen. Diese wirken darauf hin, dass die erforderlichen Leistungen oder Hilfen unverzüglich beantragt und innerhalb der Frist des $\S 14$ Abs. 2 Satz 2 erbracht werden. Die zuständige Interessenvertretung im Sinne des $\S 93$, bei schwerbehinderten Menschen außerdem die Schwerbehindertenvertretung, können die Klärung verlangen. Sie wachen darüber, dass der Arbeitgeber die ihm nach dieser Vorschrift obliegenden Verpflichtungen erfüllt.

Die Gefahr des krankheitsbedingten Arbeitsplatzverlustes soll frühzeitig erkannt und durch geeignete medizinische Maßnahmen oder Arbeitsplatzanpassung vermieden werden. Betriebliches Eingliederungsmanagement verwirklicht damit den Grundsatz „Rehabilitation vor Entlassung“. BEM setzt also gesundheitsbedingte Probleme am Arbeitsplatz voraus; sonstige betriebsbedingte Probleme sind in diesem Kontext ohne Belang. Voraussetzung für die Einleitung eines betrieblichen Eingliederungsmanagements ist die Feststellung einer Krankheit i. S. einer Diagnose nach ICD-10, die einen chronischen Verlauf genommen hat und $\mathrm{zu}$ beruflichen Teilhabestörungen führt.

Um die Ziele des BEM erreichen zu können, d.h. dass einer ,erneuten Arbeitsunfähigkeit vorgebeugt und der Arbeitsplatz erhalten werden kann“, ist die Beteiligung und ggf. Initiative des behandelnden arbeits(un)fähigkeits-beurteilenden Arztes unbedingt erforderlich, um gezielt und differenziert passende Maßnahmen ergreifen zu können (Muschalla \& Linden 2010b). Als Grundsätze gelten dabei:

- BEM betrifft nicht nur die Arbeitgeber und betroffenen Mitarbeiter, sondern muss zwingend auch die behandelnden Ärzte und Betriebsärzte mit einbeziehen.

- Eine gute Kooperation zwischen allen Beteiligten ist von großer Bedeutung.

- BEM muss als Teil eines umfassenderen Rehabilitationsproblems gesehen werden, das Krankheitsaspekte, die Arbeitsplatzsituation und die allgemeine Lebenssituation der Betroffenen gleichermaßen berücksichtigt. 
- Eine konsequente medizinische Behandlung der vorliegenden Gesundheitsstörung muss sichergestellt werden.

- Eine medizinische Beratung des Arbeitgebers und aller Beteiligten ist eine zwingende Voraussetzung für zielführende Maßnahmen.

- Rehakliniken haben die fachlichen Voraussetzungen um im Sinne eines sozialmedizinischen Konsiliardienstes bei der Problemklärung, Koordinierung, und Problemlösung mitzuwirken.

Die medizinisch-beruflich orientierte Rehabilitation (MBO) ist abzugrenzen von der „beruflichen Rehabilitation“ die sich unmittelbar auf Arbeitsförderungsmaßnahmen bezieht, also beispielsweise berufliche Ausbildungs- und Qualifizierungsmaßnahmen, finanzielle Unterstützung bei der Schaffung leidensgerechter Arbeitsplätze, Aufenthalte in Berufsförderungswerken und viele andere sog. „Leistungen zur Teilhabe am Arbeitsleben“ (LTA). Der Begriff der beruflichen Rehabilitation darf nicht mit Umschulungsleistungen gleichgesetzt werden, obwohl diese qualifizierende Bildungsleistung die bekannteste LTAMaßnahme ist. Es gibt jedoch ein sehr viel breiteres Leistungsspektrum (Hansmeier 2009, Tab. 8). Es werden Sach- und Dienstleistungen sowie unterhaltsichernde Geldleistungen unterschieden.

Tabelle 8: Leistungen zur Teilhabe am Arbeitsleben nach § 33 SGB IX

\begin{tabular}{ll}
\hline $\begin{array}{l}\text { Berufliche Rehabilitations- } \\
\text { Leistung }\end{array}$ & Beispiele \\
\hline Leistungen zur Erhaltung oder & Kraftfahrzeughilfe (Führerscheinfinanzierung, \\
Erlangung eines Arbeitsplatzes & Zuschuss zur Beschaffung eines Fahrzeugs, \\
(einschließlich Beratung und & Kostenübernahme für behinderungsbedingte \\
Vermittlung, & Zusatzausstattungen \\
Trainingsmaßnahmen, & Behindertengerechte Gestaltung von Arbeitsplätzen \\
Mobilitätshilfen) & Übernahme von Umzugskosten \\
& Arbeitsassistenz \\
\hline Berufsvorbereitung, & Blindentechnische Grundausbildung, in der bestimmte \\
\hline
\end{tabular}




\begin{tabular}{|c|c|}
\hline einschließlich der wegen einer & Grundtätigkeiten vermittelt werden: Blindenschrift und \\
\hline Behinderung erforderlichen & Umgang mit technischen Hilfen (EDV) \\
\hline \multicolumn{2}{|l|}{ Grundausbildung } \\
\hline Berufliche Anpassung, & Ausbildung: erste nach einer allgemeinen Schulbildung \\
\hline Weiterbildung, Ausbildung, & zu einem Abschluss führende Bildungsmaßnahme \\
\hline einschließlich eines zur & Berufliche Anpassung: ein medizinisch an sich \\
\hline Inanspruchnahme dieser & geeigneter Beruf ist über Jahre hinweg nicht ausgeübt \\
\hline Leistungen erforderlichen & worden und es ist eine Aufschulung auf den aktuellen \\
\hline \multirow[t]{6}{*}{ schulischen Abschlusses } & Stand erforderlich \\
\hline & Weiterbildung: Umfasst Fortbildung (Erweiterung \\
\hline & vorhandener Kenntnisse und Fähigkeiten) und \\
\hline & Umschulung (mittels eines neuen Qualifikation \\
\hline & Übergang in eine andere geeignete berufliche \\
\hline & Tätigkeit) \\
\hline \multirow[t]{6}{*}{ Überbrückungsgeld } & Grundlage ist das Arbeitsförderungsrecht. Sicherung \\
\hline & des Lebensunterhalts arbeitsloser oder von \\
\hline & Arbeitslosigkeit bedrohter Menschen oder Menschen \\
\hline & mit gesundheitlichen Beeinträchtigungen in der \\
\hline & Gründungsphase (6 Monate) einer selbstständigen \\
\hline & Existenz \\
\hline Sonstige Hilfen zur Förderung der & Kraftfahrzeughilfe, Verdienstausfall, Hilfsmittel zur \\
\hline \multirow[t]{2}{*}{ Teilhabe am Arbeitsleben } & Berufsausübung, technische Arbeitshilfen, \\
\hline & Wohnungshilfen \\
\hline \multirow[t]{5}{*}{ Leistungen an Arbeitgeber } & Eingliederungshilfen i.S. v. Probebeschäftigung, \\
\hline & Eingliederungshilfe als finanzieller Anreiz \\
\hline & Einarbeitungszuschuss bei betrieblicher Einarbeitung \\
\hline & Überbrückungshilfen bei Arbeitsaufnahme bis zur \\
\hline & ersten vollen Lohnzahlung, Fahrkostenbeihilfe \\
\hline Leistungen in Werkstätten für & Die Teilnahme an Eingangsverfahren und \\
\hline behinderte Menschen und & Arbeitstrainingsbereich soll behinderte Menschen in \\
\hline Leistungen im Eingangsverfahren & die Lage versetzen, im Arbeitsbereich einer Werkstatt \\
\hline
\end{tabular}


und im Berufsbildungsbereich für behinderte Menschen ein Mindestmaß an wirtschaftlich verwertbarer Arbeit zu verrichten

\section{Literatur}

Bundesärztekammer (2006) (Muster)Berufsordnung für die deutschen Ärztinnen und Ärzte

Beutel M, Kayser E, Vorndran A, Farley A, Bleichner F (1998) Die integrierte

berufliche Belastungserprobung in der medizinischen Rehabilitation - Erfahrungen

und Perspektiven am Beispiel der psychosomatischen Rehabilitation.

Rehabilitation 37:85-92

Dahlbender RW (2000) Psychotherapeutische Versorgungsstrukturen. In: Senf W, Broda M (Hrsg) Praxis der Psychotherapie, Thieme, Stuttgart

Deutsche Arbeitsgemeinschaft für Jugend- und Eheberatung (DAJEB) (Hrsg) (2002)

Beratungsführer. Die Beratungsstellen in Deutschland - ihre Leistungen, ihre Träger, ihre Anschriften (2 Bände). Bonn: Bundesministerium für Familie und Senioren, Eigenverlag.

Dilling H, Weyerer S, Castell R (1984) Psychische Erkrankungen in der Bevölkerung. Enke, Stuttgart

Dittmann RW, Linden M, Osterheider M, Schaaf B, Ohnmacht U, Weber HJ (2000) Antidepressant drug use: Differences between psychiatrists and general practitioners. Pharmacopsychiatry 30:28-34 
GBA Gemeinsamer Bundesausschuss der Ärzte und Krankenkassen (2004)

Richtlinien des Bundesausschusses der Ärzte und Krankenkassen über die

Beurteilung der Arbeitsfähigkeit und die Maßnahmen zur stufenweisen

Wiedereingliederung (Arbeitsunfähigkeits-Richtlinien), Bundesanzeiger 61

Hansis ML (2001) Koordinationsdefizite als Ursache vorgeworfener Behandlungsfehler,

Deutsches Ärzteblatt 98:31-32

Hansmeier T (2009) Leistungen zur Teilhabe am Arbeitleben. In Hillert A, Müller-Fahrnow W, Radoschewski FM (Hrsg) Medizinisch-berufliche orientierte Rehabilitation. Deutscher Ärzte-Verlag, Köln, 198-211

Hetzel C et al (2006) Wie lässt sich die Qualität des betrieblichen Eingliederungsmanagements messen? Die BG 11:516-519

Hiller E, Leibing E, Leichsenring F, Sulz SKD (2007) Lehrbuch Psychotherapie, Band 1

Wissenschaftliche Grundlagen der Psychotherapie. CIP Medien, München

Hillert A, Koch S, Hedlund S (2007) Stressbewältigung am Arbeitsplatz. Ein stationäres Gruppentherapieprogramm. Vandenhoeck \& Ruprecht, Göttingen

Hillert A, Müller-Fahrnow W, Radoschewski FM (2009) Medizinisch-beruflich orientierte Rehabilitation. Deutscher Ärzteverlag, Köln

Kassenärztliche Bundesvereinigung, Bundesarztregister (2001) Abrechnungsstatistik 1999. Kassenärztliche Bundesvereinigung

KBV, Kassenärztliche Bundesvereinigung (2008) Bundesarztregister, Berlin

Lamparter-Lang R (Hrsg) (1997) Patientenschulung bei chronischen Erkrankungen. Huber, Bern 
Linden M (2005) Stationäre „psychosomatische Rehabilitationen“ gemäß Sozialgesetz In: Frieboes M, Zaudig M, Nosper M (Hrsg) Rehabilitation bei psychischen Störungen. Urban und Fischer, München

Linden M (2009) Rehabilitationspsychotherapie. Definition, Aufgaben und Organisationsformen nach ICF und SGB IX. Prax Klin Verhaltensmed Rehabil 84:137-142

Linden M, Hautzinger M (Hrsg) (2008) Verhaltenstherapiemanual. Springer, Heidelberg Linden M, Muschalla B (2007) Arbeitsplatzbezogene Ängste und Arbeitsplatzphobie. Nervenarzt 78:39-44

Linden M, Maier W, Achberger M, Herr R, Helmchen H, Benkert O (1996) Psychische Erkrankungen und ihre Behandlung in Allgemeinarztpraxen in Deutschland. Ergebnisse aus einer Studie der Weltgesundheitsorganisation (WHO). Nervenarzt 67:205-215

Linden M, Leonard J, Beckerling A (2000) Behandlung depressiver Erkrankungen mit Sertralin durch Allgemeinärzte und Nervenärzte. Unterschiede und Gemeinsamkeiten. Psycho $26: 2-6$

Löffler S, Gerlich C, Lukascik M, Wolf HD, Neuderth S (2010) Praxishandbuch: Arbeits- und berufsbezogene Orientierung in der medizinischen Rehabilitation. DRV Bund, Berlin

Maier W, Linden M, Sartorius N (1996) Psychische Erkrankungen in der Allgemeinpraxis. Deutsches Ärzteblatt 93:1202-1206

Muschalla B, Linden M (2010a) Embitterment and the workplace. In Linden M, Maercker A (Hrsg) Embitterment. Societal, psychological and clinical perspectives. Springer, Wien, 152165 
Muschalla B, Linden M (2010b) Berufliches Eingliederungsmanagement und vernetzte psychosomatische Rehabilitation. In Deck R, Glaser-Möller N, Remé T. Schnittstellen der medizinischen Rehabilitation. Jacobs Verlag, Lage, 119-130

Psychotherapierichtlinien des Bundesausschusses der Ärzte und Krankenkassen in der Fassung vom 23.10.1998, in Kraft getreten am 01.01.1999. Deutsches Ärzteblatt 51-52, 1998, Ausgabe A, S. 3308-3314.

Rehabilitationszentrum Seehof (2009). Qualitätsreport 2009 Rehabilitationszentrum Seehof Teltow/Berlin. Deutsche Rentenversicherung Bund.

RKI, Robert Koch Institut und Statistisches Bundesamt (2008) Psychotherapeutische Versorgung, Heft 21, Gesundheitsberichterstattung des Bundes. Robert Koch Institut Berlin

SGB IX. Neuntes Buch Sozialgesetzbuch -Rehabilitation und Teilhabe behinderter Menschen - (Artikel 1 des Gesetzes vom 19. Juni 2001, BGBL I S. 1046), das zuletzt durch Artikel 5 des Gesetzes vom 22. Dezember 2008 geändert worden ist. http://www.juris.de

Weig W (2006) Definition der Krankenhausbehandlungsbedürftigkeit nach BSG. Nervenarzt $77: 847-851$

Wittchen HU (2000) (Hrsg) Depression 2000. Eine bundesweite Depressions-Sreening-Studie in Allgemeinarztpraxen. Fortschritte der Medizin 118:1-41

Wittchen HU (2005) Psychische Störungen in Deutschland und in der EU. Größenordnung und Belastung. Beitrag auf dem ersten deutschen Präventivkongress Dezember 2005

Wittchen HU, Müller N, Pfister H, Winter S, Schmidtkunz B (1999) Affektive, somatoforme und Angststörungen in Deutschland - Erste Ergebnisse des bundesweiten Zusatzsurveys „Psychische Störungen“. Gesundheitswesen 61:216-222

Wittchen HU, Jacobi F (2001) Die Versorgungssituation psychischer Störungen in 
Deutschland. Eine klinisch epidemiologische Abschätzung anhand des

Bundesgesundheitssurveys 1998. Bundesgesundheitsblatt, Gesundheitsforschung,

Gesundheitsschutz 44:993-1000

World Health Organization WHO (2001) International Classification of Functioning,

Disability and Health (ICF). Genf: WHO [Deutsches Institut für Medizinische Dokumentation und Information (DIMDI) (Hrsg). Die Internationale Klassifikation der Funktionsfähigkeit, Behinderung und Gesundheit - ICF. Köln: DIMDI 2004, verfügbar unter URL: www.dimdi.de]

\section{Multiple Choice Fragen}

1) Wie hoch ist der Anteil an Patienten mit psychischen Erkrankungen in Allgemeinarztpraxen?
(A) $5 \%$
(B) $15 \%$
(C) $25 \%$
(D) $35 \%$
(E) $45 \%$

2) Was gibt es bei der Richtlinienpsychotherapie nicht?
(A) Erstzugangsrecht
(B) freie Therapeutenwahl
(C) Beantragung
(D) Psychotherapie als Krankenbehandlung
(E) allgemeine Lebensberatung

3) Welches ist kein Kriterium für die Bewilligung einer Richtlinienpsychotherapie?
(A) Notwendigkeit
(B) Zweckmäßigkeit
(C) Wirtschaftlichkeit
(D) Prognose
(E) Neuartigkeit

4) Wo ist die Maßnahme zum „Betrieblichen Eingliederungsmanagement“ geregelt? 

(A) § 84 SGB IX
(B) $\S 51 \mathrm{SGB} V$
(C) $\S 74$ SGB V
(D) $\S 28$ SGB IX
(E) $\S 111$ SGB V

5) Eine Patientin mit einer seit mehreren Wochen bestehenden depressiven Symptomatik stellt sich vor: Der Befund ergibt einen reduzierten Affekt, Insuffizienzerleben, Grübelneigung, erhaltene Konzentrationsfähigkeit und leicht reduziertem Antrieb. Sie sagt es gehe ihr so schlecht, in ihrem Beruf als Krankenschwester könne sie so nicht mehr weiterarbeiten. Welche Maßnahme ist am ehesten indiziert?
(A) Ausstellung einer Arbeitsunfähigkeitsbescheinigung
(B) Antrag auf medizinische Rehabilitation
(C) Antrag auf berufliche Rehabilitation
(D) Einleitung einer Richtlinienpsychotherapie
(E) Klärung des konkreten Arbeitsplatzprofils und Leistungsanforderungen

6) Die Dokumentation von Psychotherapie ist für den behandelnden Therapeuten in erster Linie bedeutsam
(A) für Rentenanträge
(B) zur Absicherung gegen Schadensersatzansprüche von Patienten
(C) bei Klärung von Haftpflichtfragen
(D) bei der Fortsetzung der Behandlung durch Andere
(E) für die Stundenstrukturierung

7) Was gilt nicht bei der Aushändigung von Krankenunterlagen an den Patienten?

\section{(A) Das schützenswerte Interesse des Patienten an ihn betreffenden Daten überwiegt nicht die Persönlichkeitsrechte des Therapeuten}

(B) Eine Unterteilung in einen objektiven und subjektiven Teil der Krankengeschichte hat in der Regel keinen Bestand

(C) Es sind schutzwürdige Interessen Dritter zu beachten

(D) Es gilt das Recht des Patienten auf Selbstbestimmung

(E) Es gilt das Recht auf Akteneinsicht für den Patienten

8) Für die ambulante Behandlung von Patienten der Gesetzlichen Krankenversicherung gilt/gelten ...
(A) der Einheitliche Bewertungsmaßstab (EBM)
(B) die Gebührenordnung für Ärzte (GOÄ)
(C) die Psychotherapiehonorar-Ordnung
(D) die Beihilfevorschriften
(E) die Gebührenordnung für Psychotherapeuten (GOP) 
9) Wer ist Kostenträger für eine „Stufenweise Wiedereingliederung“ direkt im Anschluss an eine stationäre psychosomatische Rehabilitationsbehandlung?
(A) Arbeitsamt
(B) Rentenversicherung
(C) Krankenkasse
(D) Arbeitgeber
(E) Integrationsamt

10) Was ist keine Maßnahme im Rahmen einer „Beruflichen Rehabilitation“?
(A) Ausbildungsmaßnahme
(B) Qualifizierungsmaßnahme
(C) Gruppentraining Stressbewältigung am Arbeitsplatz im Rahmen einer stationären medizinischen Rehabilitation
(D) Finanzieller Zuschuss zur Schaffung eines leidensgerechten Arbeitsplatzes
(E) Durchführung einer Maßnahme in einem Berufsförderungswerk oder
Berufstrainingszentrum 